\title{
Generalized Swanson Models and their solutions
}

\author{
A. Sinhd 1 \\ and \\ P. Roy 2 \\ Physics 8 Applied Mathematics Unit \\ Indian Statistical Institute \\ Kolkata - 700108 \\ INDIA
}

\begin{abstract}
We analyze a class of non-Hermitian quadratic Hamiltonians, which are of the form $H=\mathcal{A}^{\dagger} \mathcal{A}+\alpha \mathcal{A}^{2}+\beta \mathcal{A}^{\dagger}$, where $\alpha, \beta$ are real constants, with $\alpha \neq \beta$, and $\mathcal{A}^{\dagger}$ and $\mathcal{A}$ are generalized creation and annihilation operators. Thus these Hamiltonians may be classified as generalized Swanson models. It is shown that the eigenenergies are real for a certain range of values of the parameters. A similarity transformation $\rho$, mapping the non-Hermitian Hamiltonian $H$ to a Hermitian one $h$, is also obtained. It is shown that $H$ and $h$ share identical energies. As explicit examples, the solutions of a couple of models based on the trigonometric RosenMorse I and the hyperbolic Rosen-Morse II type potentials are obtained. We also study the case when the non-Hermitian Hamiltonian is $\mathcal{P} \mathcal{T}$ symmetric.
\end{abstract}

PACS : 03.65-w

Key words : Generalized Swanson model, non-Hermitian Hamiltonian, $\eta$-pseudo Hermiticity, similarity transformation, Rosen Morse potential

\footnotetext{
${ }^{1}$ e-mail : anjana_t@isical.ac.in

${ }^{2} \mathrm{e}-\mathrm{mail}$ : pinaki@isical.ac.in
} 


\section{Introduction}

The generalization of standard quantum mechanics and quantum field theory to include complex or non-Hermitian potentials with real spectrum, has been intensively studied during the last few years [1, 2, 3, 4], primarily because of their immense potential for possible applications in a wide range of phenomena, e.g., nuclear physics [5], scattering theory (i.e., complex absorbing potentials) [6], field theory [7], periodic potentials [9], quantum cosmology [8], random matrix theory [10], etc. Initially, the reality of the spectrum was attributed to the so-called $\mathcal{P} \mathcal{T}$ symmetry of the system, i.e.,

$$
H \neq H^{\dagger} \quad, \quad H \mathcal{P} \mathcal{T}=\mathcal{P} \mathcal{T} H
$$

where $\mathcal{P}$ stands for parity and $\mathcal{T}$ denotes time reversal operators respectively

$$
\mathcal{P} x \mathcal{P}=-x, \quad \mathcal{P} p \mathcal{P}=\mathcal{T} p \mathcal{T}=-p, \quad \mathcal{T}(i .1) \mathcal{T}=-i .1
$$

Such Hamiltonians were found to possess a real and discrete spectrum when $\mathcal{P} \mathcal{T}$ symmetry is exact, i.e., the energy eigenstates are also the eigenstates of $\mathcal{P} \mathcal{T}$; if not then $\mathcal{P} \mathcal{T}$ symmetry is said to be spontaneously broken and the energies occur as complex conjugate pairs.

However, it was soon discovered that $\mathcal{P} \mathcal{T}$ symmetry is neither the necessary nor the sufficient criterion for the spectrum to be real. Subsequent works showed that the necessary and sufficient condition for a non-Hermitian Hamiltonian to possess real and discrete spectrum, is its $\eta$-pseudo Hermiticity, such that $H$ are linear operators acting in a Hilbert space (generally different from the physical Hilbert space), and satisfying [11] :

$$
H^{\dagger}=\eta H \eta^{-1} \text {, i.e. } H^{\dagger} \eta=\eta H
$$

where $\eta$ is a linear, Hermitian, invertible operator. It may be mentioned that for a given pseudo-Hermitian operator $H$, the metric operator $\eta$ is not unique. Furthermore, the pseudo-Hermiticity of $H$ is equivalent to the presence of an antilinear symmetry, $\mathcal{P} \mathcal{T}$ symmetry being the primary example [12]. Conversely, a quantum system possessing an exact antilinear symmetry is pseudo-Hermitian, and is equivalent to a quantum system described by a Hermitian Hamiltonian $h$. Thus $H$ may be mapped to $h$, by a similarity transformation $\rho$ [11, 13]. For example, let an eigenvalue (Sturm-Liouville) equation or a differential operator $H$ act in a complex function space $\mathcal{V}$, endowed with a positive definite inner product, such that it is described by the Hilbert space $\mathcal{H}$. In such a case there exists a mapping from the non-Hermitian $H$ to its Hermitian counterpart $h$, through a similarity transformation $\rho$ [14]; i.e.,

$$
h=\rho H \rho^{-1}
$$

with $\rho$ being the unique positive-definite square root of $\eta$ :

$$
\rho=\sqrt{\eta}
$$


A relation similar to (44) holds for observables as well. For example, if $\mathcal{O}_{h}$ is an observable in the Hermitian theory described by $h$, then the corresponding observable in the pseudoHermitian theory is given by

$$
\mathcal{O}=\rho^{-1} \mathcal{O}_{h} \rho
$$

Though known for a long time [15], the idea of pseudo Hermiticity was revived after the concept of $\mathcal{P} \mathcal{T}$ symmetry was introduced a decade ago.

Recently, Swanson analyzed the real but non-Hermitian, $\mathcal{P} \mathcal{T}$ symmetric quadratic Hamiltonian [16]

$$
H=\omega a^{\dagger} a+\alpha a^{2}+\beta a^{\dagger} \quad, \quad \alpha \neq \beta
$$

where $a^{\dagger}, a$ are the Harmonic oscillator creation and annihilation operators for unit frequency,

$$
a=\frac{d}{d x}+x \quad, \quad a^{\dagger}=-\frac{d}{d x}+x
$$

and $\omega, \alpha, \beta$ are real parameters with dimensions of inverse time. It was shown that for $\alpha \neq \beta$, though the Hamiltonian $H$ is non-Hermitian, yet the eigenvalues were real and positive for $\omega^{2} \geq 4 \alpha \beta$. This model has attracted the attention of several workers in recent times, e.g. [17, 18]. In this work, we focus our attention on the pseudo-Hermitian generalization of the Swanson model (7), which may not necessarily be $\mathcal{P} \mathcal{T}$ symmetric. The simplest and most straightforward generalization would be to consider generalized creation and annihilation operators $\mathcal{A}^{\dagger}$ and $\mathcal{A}$ in place of $a^{\dagger}$ and $a$, of the form

$$
\begin{aligned}
\mathcal{A} & =\frac{d}{d x}+W(x) \\
\mathcal{A}^{\dagger} & =-\frac{d}{d x}+W(x)
\end{aligned}
$$

The function $W(x)$, called the pseudo superpotential (in analogy with conventional supersymmetry), given by

$$
W(x)=-\frac{f_{0}{ }^{\prime}(x)}{f_{0}(x)}
$$

where $f_{0}(x)$ is the ground state wave function of the Schrödinger Hamiltonian $\mathbf{H}=\mathcal{A}^{\dagger} \mathcal{A}$. For the particular case of $W(x)$ being a linear function in $x$, we get back the Swanson Hamiltonian in (7). This is somewhat analogous to the generalization of the JaynesCummings model to other two level shape-invariant bound state systems [19], applying the principles of supersymmetric quantum mechanics [20]. Thus our starting Hamiltonian would be

$$
H=\mathcal{A}^{\dagger} \mathcal{A}+\alpha \mathcal{A}^{2}+\beta \mathcal{A}^{\dagger}{ }^{2}, \quad \alpha \neq \beta
$$

where $\alpha, \beta$ are real, dimensionless constants. Obviously the model given in (11) above, is non-Hermitian for $\alpha \neq \beta$. In particular, our attempt will be to give the general formalism for solving such a non-Hermitian Hamiltonian, and examine the range of values of the 
parameters for which the energies are real. This situation is similar to [16], where real energies were found only when the parameters satisfied certain constraints. On the other hand since the Hamiltonian $H$ does not admit real energies for arbitrary values of the parameters, the model can be termed as conditionally exactly solvable (CES) [21]. We shall restrict our study to $\eta$ pseudo-Hermitian Hamiltonians only, as $\eta$-pseudo Hermiticity is the necessary and sufficient condition for the existence of real energies. We shall also find a similarity transformation $\rho$, mapping the non-Hermitian Hamiltonian $H$ to the Hermitian one $h$, for a certain class of models. It will be shown that $H$ and $h$ share identical energies. It may be mentioned here that though the existence of $\eta$, and hence $\rho$, is guaranteed, it may not always be possible to determine the Hermitian counterpart $h$ exactly. For example, the relationship between the non-Hermitian $H$ and its hermitian entity $h$, was explored in [22], for the Swanson model [16] and the $i g x^{3}$ potential. However, in the first case, $h$ turned out to be a scaled harmonic oscillator, while in the second model $h$ could be constructed perturbatively only. It may be mentioned here that the operator method was employed in [16] while we work with the differential equation directly. The simplicity of the present formalism lies in the fact that $h$ can be determined in a straightforward manner, and secondly, $\rho$, and hence $\eta$, can be found exactly, for the class of non-Hermitian models considered in this work.

The organization of the paper is as follows. In section 2, we shall give the general formalism for solving a class of non-Hermitian Swanson model with generalized creation and annihilation operators. The similarity transformation $\rho$, between the Hermitian $h$

and the non-Hermitian $H$, is established in section 3, while the pseudo Hermiticity of $H$ is shown in section 4 . We illustrate our results with the help of a couple of explicit examples in sections 5 and 6, with Hamiltonians based on the trigonometric Rosen-Morse I and the hyperbolic Rosen-Morse II potentials, respectively. In section 7, a special subclass of pseudo-Hermitian Hamiltonians are considered, which are $\mathcal{P} \mathcal{T}$ symmetric as well. Finally, section 8 is kept for Conclusions and Discussions.

\section{Theory}

As mentioned above, we shall examine a generalization of the Swanson model , viz., [16]

$$
H=\mathcal{A}^{\dagger} \mathcal{A}+\alpha \mathcal{A}^{2}+\beta \mathcal{A}^{\dagger 2}, \quad \alpha \neq \beta
$$

where $\alpha$ and $\beta$ are constants, dimensionless as well as real. Evidently, $H$ is non-Hermitian for $\alpha \neq \beta$ for any real $W(x)$. With the help of (9), the eigenvalue equation corresponding 
to (11) reads

$$
\begin{aligned}
H \psi & =\left\{-(1-\alpha-\beta) \frac{d^{2}}{d x^{2}}+2(\alpha-\beta) W \frac{d}{d x}+(1+\alpha+\beta) W^{2}-(1-\alpha+\beta) W^{\prime}\right\} \psi \\
& =\left\{-(1-\alpha-\beta)\left(\frac{d}{d x}-\frac{\alpha-\beta}{1-\alpha-\beta} W\right)^{2}+\frac{1-4 \alpha \beta}{(1-\alpha-\beta)} W^{2}-W^{\prime}\right\} \psi \\
& =E \psi
\end{aligned}
$$

The term $\left(-\frac{\alpha-\beta}{1-\alpha-\beta} W(x)\right)$ in the parenthesis takes the form of a complex vector potential and can be eliminated by a gauge transformation of the form [23]

$$
\psi(x)=e^{\mu \int W(x) d x} \phi(x) \quad, \quad \text { with } \quad \mu=\frac{\alpha-\beta}{1-\alpha-\beta}, \alpha+\beta \neq 1
$$

Thus (12) reduces to the well known Schrödinger form

$$
h \phi(x)=\left(-\frac{d^{2}}{d x^{2}}+V(x)\right) \phi(x)=\varepsilon \phi(x)
$$

where

$$
\begin{aligned}
V(x) & =\left(\frac{\sqrt{1-4 \alpha \beta}}{1-\alpha-\beta} W(x)\right)^{2}-\frac{1}{(1-\alpha-\beta)} W^{\prime}(x) \\
\varepsilon & =\frac{E}{1-\alpha-\beta}
\end{aligned}
$$

It is well known from supersymmetric quantum mechanics [20], that $h$ can always be written in a factorizable form as a product of a pair of linear differential operators $A, A^{\dagger}$, as

$$
\begin{aligned}
h & =A^{\dagger} A+\epsilon \\
& =-\frac{d^{2}}{d x^{2}}+\mathrm{w}^{2}-\mathrm{w}^{\prime}+\epsilon
\end{aligned}
$$

where $\epsilon$ is the factorization energy, and $A, A^{\dagger}$ and w $(x)$ are given by

$$
A=\frac{d}{d x}+\mathrm{w}(x), \quad A^{\dagger}=-\frac{d}{d x}+\mathrm{w}(x), \quad \mathrm{w}(x)=-\frac{d \ln \varphi_{0}(x)}{d x}
$$

Here $\varphi_{0}$ is the ground state eigenfunction of $A^{\dagger} A$ with energy $\varepsilon_{0}$. It may be mentioned here that SUSY is said to be unbroken when the ground state energy $\varepsilon_{0}=0$. 
Evidently, if we can identify the term $V(x)$ in (15) above, with an exactly solvable potential, then we can easily find the solutions of $h$. To this end, for further convenience, $V(x)$ can be identified with a shape-invariant potential, as using the ideas of supersymmetric quantum mechanics [20], the raising and lowering operator method of harmonic oscillator can be generalized to a whole class of shape invariant potentials [24], which includes all the analytically solvable models. To narrow down the class of potentials further, our strategy would be to write $V(x)$ in (15) in the supersymmetric form $\mathrm{w}^{2}(x)-\mathrm{w}^{\prime}(x)$ as given in (16). This identification enables us to find the energies $(E)$ and the eigenfunctions $(\psi)$ of the eigenvalue equation in (12). However, this imposes certain restrictions on the permissible values of $\alpha$ and $\beta$. For real energies, supersymmetric considerations require the term containing $W^{2}(x)$ in the expression for $V(x)$ in (15), must be positive. Furthermore, $E$ and $\varepsilon$ should have similar behaviour. Hence, the parameters $\alpha, \beta$ must satisfy the following constraints, irrespective of the explicit form of $W(x)$ :

$$
\alpha+\beta<1 \quad, \quad 4 \alpha \beta<1
$$

In addition to the general restrictions imposed on $\alpha, \beta$ in (18), there may be some more constraints depending on the particular choice of the model, arising from the normalizability requirement of the wave functions. We shall illustrate our observations with the help of a couple of explicit examples in the next section. The fact that both the models considered here are pseudo-Hermitian will be shown in a later section.

\section{Similarity Transformation between $H$ and $h$}

In this section we shall determine a similarity transformation, mapping the non-Hermitian $H$ to the Hermitian $h$ [11]. For this purpose we focus our attention on the gauge transformation $\rho$ relating $\psi(x)$ and $\phi(x)$ in equation (13); i.e.,

$$
\rho=e^{-\mu \int W d x} \quad, \quad \mu=\frac{\alpha-\beta}{1-\alpha-\beta}
$$

where $W(x)=-\frac{f_{0}{ }^{\prime}(x)}{f_{0}(x)}, f_{0}(x)$ being the ground state wave function of the Schrödinger Hamiltonian $\mathbf{H}=\mathcal{A}^{\dagger} \mathcal{A}$. Let $\psi(x)$ be an eigenfunction of $H$, with eigenvalue $E$ :

$$
H \psi=E \psi
$$

Let us now apply the transformation $\rho$ to the above eigenfunction $\psi(x)$; i.e.,

$$
\phi(x)=\rho \psi(x)
$$


Then (20) can be written as

$$
H \rho^{-1} \phi(x)=E \rho^{-1} \phi(x) \quad \text { or } \quad \rho H \rho^{-1} \phi(x)=E \phi(x)
$$

Thus $\phi(x)$ is a solution of the equation $h \phi=E \phi$ with the same energy $E$ as in (20), provided $H$ is mapped to $h$ by the similarity transformation in (4), viz.,

$$
h=\rho H \rho^{-1}
$$

As we have observed in this work earlier, $h$ is Hermitian, though $H$ is non-Hermitian. Thus the similarity transformation $\rho$ given in (19) maps the pseudo-Hermitian Hamiltonian $H$ in the generalized version of the Swanson model to its Hermitian counterpart $h$. Furthermore, this exact form of the similarity operator for this class of models, also gives the wavefunctions in the corresponding Hermitian picture. This will be clarified further by the explicit models discussed later in this work.

\section{Pseudo Hermiticity of $H$}

We shall show in this section that, although $H$ in (12) is non $\mathcal{P} \mathcal{T}$ symmetric, it is in fact, pseudo-Hermitian, with respect to a linear, invertible, Hermitian operator $\eta$, and that it is in fact the square of the similarity transformation $\rho$, i.e., $\eta=\rho^{2}$.

We start with the eigenvalue equation $H \psi=E \psi$, where

$$
\begin{aligned}
H & =\mathcal{A}^{\dagger} \mathcal{A}+\alpha \mathcal{A}^{2}+\beta \mathcal{A}^{\dagger 2} \\
& =-(1-\alpha-\beta)\left(\frac{d}{d x}-\frac{\alpha-\beta}{1-\alpha-\beta} W(x)\right)^{2}+\frac{1-4 \alpha \beta}{1-\alpha-\beta} W^{2}(x)-W^{\prime}(x)
\end{aligned}
$$

Now, let us explore the relationship between $H$ and its adjoint $H^{\dagger}$, given by

$$
\begin{aligned}
H^{\dagger} & =\mathcal{A}^{\dagger} \mathcal{A}+\alpha \mathcal{A}^{\dagger 2}+\beta \mathcal{A}^{2} \\
& =-(1-\alpha-\beta)\left(\frac{d}{d x}+\frac{\alpha-\beta}{1-\alpha-\beta} W(x)\right)^{2}+\frac{1-4 \alpha \beta}{1-\alpha-\beta} W^{2}(x)-W^{\prime}(x)
\end{aligned}
$$

If we put

$$
\eta=\rho^{2}=e^{-2 \mu \int W d x}, \quad \mu=\frac{\alpha-\beta}{1-\alpha-\beta}
$$

then it can be shown by straightforward calculations that $H$ and $H^{\dagger}$ are related by (3), viz.,

$$
H^{\dagger} \eta=\eta H \quad \text { i.e., } \quad H^{\dagger}=\eta H \eta^{-1}
$$


In other words, $H$ respects the condition for pseudo Hermiticity [11]. Thus this approach enables us to determine the exact form of the pseudo Hermiticity operator $\eta$, which in turn, is related to the similarity transformation $\rho=\sqrt{\eta}$.

\section{A Model based on Trigonometric Rosen-Morse I Potential}

The trigonometric Rosen-Morse I model [20] is described by the potential

$$
V(x)=A(A-1) \csc ^{2} x+2 B \cot x-A^{2}+\frac{B^{2}}{A^{2}}, \quad 0 \leq x \leq \pi
$$

In the language of supersymmetry, if the potential in (25) can be written in terms of a superpotential $\mathrm{w}(x)$ as

$$
V(x)=\mathrm{w}^{2}(x)-\mathrm{w}^{\prime}(x)
$$

then a suitable ansatz of $\mathrm{w}(x)$ may be given by

$$
\mathrm{w}(x)=-A \cot x-\frac{B}{A} \quad, \quad A>0, B>0
$$

For our model, keeping analogy with the above, we consider the following form of the function $W(x)$, in the construction of the generalized annihilation and creation operators $\mathcal{A}$ and $\mathcal{A}^{\dagger}$ in (9) :

$$
W(x)=-A_{1} \cot x-\frac{B_{1}}{A_{1}} \quad, \quad A_{1}>0, B_{1}>0
$$

Obviously, the Hamiltonian in (12) constructed from this $W(x)$ is non-Hermitian (as well as non $\mathcal{P} \mathcal{T}$ symmetric ) for $\alpha \neq \beta$. Substitution of (28) in (13) yields

$$
\psi(x)=e^{-\mu_{1} x} \sin ^{\mu_{2}} x \quad \phi(x)
$$

where

$$
\mu_{1}=\frac{B_{1}}{A_{1}} \frac{(\alpha-\beta)}{(1-\alpha-\beta)}, \quad \mu_{2}=-\frac{A_{1}(\alpha-\beta)}{(1-\alpha-\beta)}
$$

Now, we are interested in real energies only. Additionally, the wavefunctions must satisfy certain boundary conditions, e.g., well behaved behaviour at the boundaries $x \rightarrow 0$ and $x \rightarrow \pi$, and normalizability requirement. So $\mu_{2}>0$. These impose further restrictions on $\alpha$ and $\beta$, so that they must obey the following condition :

$$
\alpha<\beta
$$


Thus (14) reduces to the trigonometric Rosen Morse I model in (25), with potential

$$
V(x)=\sigma \csc ^{2} x+2 B_{1} \frac{1-4 \alpha \beta}{(1-\alpha-\beta)^{2}} \cot x\left(A_{1}^{2}-\frac{B_{1}^{2}}{A_{1}^{2}}\right) \frac{1-4 \alpha \beta}{(1-\alpha-\beta)^{2}}
$$

where

$$
\sigma=\frac{A_{1}^{2}(1-4 \alpha \beta)-A_{1}(1-\alpha-\beta)}{(1-\alpha-\beta)^{2}}
$$

so that $A$ and $B$ can be identified with

$$
A=\frac{1}{2} \pm \frac{\sqrt{1+4 \sigma}}{2} \quad, \quad B=B_{1} \frac{1-4 \alpha \beta}{(1-\alpha-\beta)^{2}}
$$

Since $A>0$, only the positive sign is allowed in the expression for $A$ in (34). Moreover, as is obvious from (32), for the existence of bound states, $\sigma>0$. Since $A_{1} \neq 0$, hence this condition requires

$$
A_{1}>\frac{1-\alpha-\beta}{1-4 \alpha \beta}
$$

The energy eigenvalues and the corresponding eigenfunctions of (25) are well known [20]

$$
\epsilon_{n}=(A+n)^{2}-\frac{B^{2}}{(A+n)^{2}}-A^{2}+\frac{B^{2}}{A^{2}} \quad, \quad n=0,1,2, \cdots
$$

Therefore (32) has solutions

$$
\varepsilon_{n}=(A+n)^{2}-\frac{B^{2}}{(A+n)^{2}}-\left(A_{1}^{2}-\frac{B_{1}^{2}}{A_{1}^{2}}\right) \frac{1-4 \alpha \beta}{(1-\alpha-\beta)^{2}}
$$

where $A$ and $B$ are given in terms of $A_{1}$ and $B_{1}$ through (34), and the wavefunctions are

$$
\begin{gathered}
\phi_{n}(x) \approx\left(y^{2}-1\right)^{-\frac{(A+n)}{2}} e^{\left(\frac{B}{A+n}\right) x} P_{n}^{\left(s_{+}, s_{-}\right)}(y), \quad y=i \cot x \\
s_{ \pm}=-A-n \pm i \frac{B}{(A+n)}
\end{gathered}
$$

In (38) above, $P_{n}^{\left(s_{+}, s_{-}\right)}(y)$ are the standard Jacobi polynomials [25]. Using (37) and (38) one can easily obtain the energies and eigenfunctions of the eigenvalue equation in (12), for this particular model as :

$$
\begin{gathered}
E_{n}=(1-\alpha-\beta) \varepsilon_{n} \\
\psi_{n}(x) \approx e^{\left\{\frac{B}{(A+n)}-\mu_{1}\right\} x} \sin ^{A+n+\mu_{2}} x P_{n}^{\left(s_{+}, s_{-}\right)}(y), \quad y=i \cot x
\end{gathered}
$$

Thus one gets the complete solution of the non-Hermitian Hamiltonian in (11), by reducing it to the corresponding Hermitian system. 


\section{Choice of parameters}

To show that the solutions (40) and (41) actually exist, it is necessary to show that there are parameter values actually satisfying (18), (31) and (35). There may be innumerable such combinations of $\alpha, \beta, A_{1}$ and $B_{1}$. We show a few possible values of these parameters in Table 1. In each case, the potential is given as in (32), with solutions $\psi_{n}(x)$ given in (41) above, and energies in (40).

Table 1 : Some values of the parameters for the model with $W(x)$ as given in (28)

\begin{tabular}{|c|c|c|c|c|c|c|c|c|c|c|c|}
\hline$\alpha$ & $\beta$ & $\alpha+\beta$ & $4 \alpha \beta$ & $A_{1}$ & $B_{1}$ & $\mu_{1}$ & $\mu_{2}$ & $\sigma$ & $A$ & $B$ & $E_{n}$ \\
\hline $1 / 4$ & $1 / 2$ & $3 / 4$ & $1 / 2$ & $3 / 2$ & $1 / 8$ & $-1 / 12$ & $3 / 2$ & 12 & 4 & 1 & $\frac{1}{4} \varepsilon_{n}$ \\
\hline $1 / 4$ & $2 / 3$ & $11 / 12$ & $2 / 3$ & 1 & $1 / 2$ & $-5 / 2$ & 5 & 36 & 6.52 & 24 & $\frac{1}{12} \varepsilon_{n}$ \\
\hline $1 / 8$ & $3 / 4$ & $7 / 8$ & $3 / 8$ & 1 & 2 & -10 & 5 & 32 & 6.18 & 80 & $\frac{1}{8} \varepsilon_{n}$ \\
\hline $1 / 3$ & $1 / 2$ & $5 / 6$ & $2 / 3$ & 1 & 2 & -2 & 1 & 6 & 3 & 36 & $\frac{1}{6} \varepsilon_{n}$ \\
\hline
\end{tabular}

One can check the nature of the non Hermitian Hamiltonian and the corresponding Hermitian equivalent for this model. For example, for the values of parameters in the first line of Table 1, the starting non-Hermitian equation (12), is given by

$$
\begin{aligned}
H \psi(x) & =\left\{-\frac{1}{4} \frac{d^{2}}{d x^{2}}+\left(\frac{18 \cot x+1}{24}\right) \frac{d}{d x}+\frac{33}{16}(\csc x)^{2}+\frac{7}{16} \cot x-\frac{2261}{576}\right\} \psi(x) \\
& =E \psi(x)
\end{aligned}
$$

With the help of the similarity transformation in (19), the above non-Hermitian equation is transformed to the Hermitian one

$$
h \phi(x)=\left\{-\frac{d^{2}}{d x^{2}}+12 \csc ^{2} x+2 \cot x-\frac{323}{18}\right\} \phi(x)=\varepsilon \phi(x)
$$

where $E=\frac{1}{4} \varepsilon$, and $\psi$ and $\phi$ are related by

$$
\psi(x)=e^{\frac{1}{12} x} \sin ^{\frac{3}{2} x} \phi(x)
$$

Since equation (43) can be solved exactly, one can use its solutions to find the energies and eigenfunctions of the non-Hermitian equation in (42).

It is worth mentioning here that a second order linear differential equation can have only two linearly independent solutions. For the model discussed in this section, only 
one of the solutions is normalizable in the Hermitian picture. So the second solution is not considered. It can be checked by straightforward algebra that even when they are mapped to the non Hermitian picture, the second solution does not have well defined behaviour at the boundaries, irrespective of the fact whether the parameters $\alpha, \beta$ obey the constraints (18) or not. Furthermore, for the acceptable set of solutions in the Hermitian picture, well-defined behaviour of the eigenfunctions at the boundaries, and the normalization condition, hold only when the parameters $\alpha, \beta$, etc. satisfy the constraints (18), (27), (31) and (35). Detailed but simple calculations reveal that the constraints remain unaltered when one moves from the Hermitian to the non Hermitian picture. Hence the solutions given here represent the complete set, in both the Hermitian as well as the non Hermitian picture.

\section{A Model based on Hyperbolic Rosen-Morse II Po- tential}

As a second non-Hermitian as well as non $\mathcal{P} \mathcal{T}$ symmetric example, we shall consider a model based on the hyperbolic Rosen-Morse II potential, given by [20]

$$
\begin{aligned}
V(x) & =-a(a+1) \operatorname{sech}^{2} x+2 b \tanh x+a^{2}+\frac{b^{2}}{a^{2}}, \quad b<a^{2},-\infty \leq x \leq \infty \\
& =\mathrm{w}^{2}(x)-\mathrm{w}^{\prime}(x)
\end{aligned}
$$

with the superpotential $\mathrm{w}(x)$ of the form

$$
\mathrm{w}(x)=a \tanh x+\frac{b}{a}, \quad b<a^{2} \quad \text { and } \quad \mathrm{a}, \mathrm{b}>0
$$

Analogous to the previous example, to construct the generalized annihilation and creation operators in (9), we take the following ansatz for $W(x)$ :

$$
W(x)=A_{2} \tanh x+\frac{B_{2}}{A_{2}}, \quad B_{2}<A_{2}^{2} \quad \text { and } \quad \mathrm{A}_{2}, \mathrm{~B}_{2}>0
$$

Proceeding along the lines similar to the earlier example, the eigenvalue equation in (14) reduces to that of the well-known hyperbolic Rosen Morse-II model in (45), with the potential

$$
V(x)=-\chi \operatorname{sech}^{2} x+2 B_{2} \frac{1-4 \alpha \beta}{(1-\alpha-\beta)^{2}} \tanh x+\left(A_{2}^{2}+\frac{B_{2}^{2}}{A_{2}^{2}}\right) \frac{1-4 \alpha \beta}{(1-\alpha-\beta)^{2}}
$$


provided one makes the identification

$$
a=-\frac{1}{2} \pm \frac{\sqrt{1+4 \chi}}{2}, \quad b=B_{2} \frac{1-4 \alpha \beta}{(1-\alpha-\beta)^{2}}
$$

with

$$
\chi=\frac{A_{2}^{2}(1-4 \alpha \beta)+A_{2}(1-\alpha-\beta)}{(1-\alpha-\beta)^{2}}
$$

Once again, since $a>0$, only the positive sign is allowed in (49) in the expression for $a$. Thus the solutions $\phi(x)$ of the eigenvalue equation in (14) with the potential in (48), are related to the solutions $\psi(x)$ of $H$ in (12) by the substitution in (13) :

$$
\psi(x)=e^{\mu_{1} x} \cosh ^{\mu_{2}} x \phi(x)
$$

with

$$
\mu_{1}=\frac{B_{2}}{A_{2}} \frac{(\alpha-\beta)}{(1-\alpha-\beta)}, \quad \mu_{2}=\frac{A_{2}(\alpha-\beta)}{(1-\alpha-\beta)}, \quad \alpha+\beta<1
$$

For the eigenfunction to be well behaved at $x= \pm \infty, \mu_{2}$ should be negative, so that $\alpha<\beta$. Additionally, $\left|\mu_{2}\right|>\left|\mu_{1}\right|$, which, in turn, requires $B_{2}<A_{2}^{2}$, as already mentioned in (47). These constraints on $\alpha, \beta$, which depend on the explicit form of the model considered, are in addition to the ones in (18). The energy eigenvalues and eigenfunctions to (48) are respectively given by,

$$
\begin{gathered}
\varepsilon_{n}=-(a-n)^{2}-\frac{b^{2}}{(a-n)^{2}}+\left(A_{2}^{2}+\frac{B_{2}^{2}}{A_{2}^{2}}\right) \frac{1-4 \alpha \beta}{(1-\alpha-\beta)^{2}} \quad, \quad n<a \\
\phi_{n}(x) \approx(1-y)^{s_{+} / 2}(1+y)^{s_{-} / 2} P_{n}^{\left(s_{+}, s_{-}\right)}(y), \quad y=\tanh x
\end{gathered}
$$

where

$$
s_{ \pm}=a-n \pm \frac{b}{a-n}
$$

$P_{n}^{\left(s_{+}, s_{-}\right)}(y)$ are the Jacobi polynomials [25], and $a, b$ are given in terms of $A_{2}, B_{2}$ through (49) and (50). The corresponding energies and the eigenfunctions of the eigenvalue equation in (12) are obtained as :

$$
\begin{gathered}
E_{n}=(1-\alpha-\beta) \varepsilon_{n} \quad, \quad n=0,1,2, \cdots<a \\
\psi_{n}(x) \approx(1-y)^{\left(s_{+}-\mu_{2}\right) / 2}(1+y)^{\left(s_{-}-\mu_{2}\right) / 2} e^{\mu_{1} x} P_{n}^{\left(s_{+}, s_{-}\right)}(y), \quad y=\tanh x
\end{gathered}
$$

For normalizable functions with real energies, and well defined behaviour at $x \rightarrow \pm \infty$, the constraints given in (31) hold here, too.

\section{Choice of parameters}

Analogous to the previous case, here, too, the solutions (56) and (57) are acceptable in certain ranges of the parameters $\alpha, \beta$, satisfying (18) and (31). Many such combinations are possible. We list a few cases in Table 2 . 
Table 2 : Some values of the parameters for the model with $W(x)$ as given in (47)

\begin{tabular}{|c|c|c|c|c|c|c|c|c|c|c|c|}
\hline$\alpha$ & $\beta$ & $\alpha+\beta$ & $4 \alpha \beta$ & $A_{2}$ & $B_{2}$ & $\mu_{1}$ & $\mu_{2}$ & $\chi$ & $a$ & $b$ & $E_{n}$ \\
\hline $1 / 4$ & $1 / 2$ & $3 / 4$ & $1 / 2$ & $3 / 2$ & $1 / 4$ & $-1 / 6$ & $-3 / 2$ & 24 & 4.42 & 2 & $\frac{1}{4} \varepsilon_{n}$ \\
\hline $1 / 3$ & $1 / 2$ & $5 / 6$ & $2 / 3$ & 1 & $1 / 8$ & $-1 / 8$ & -1 & 18 & 3.74 & $3 / 2$ & $\frac{1}{6} \varepsilon_{n}$ \\
\hline $1 / 6$ & $1 / 3$ & $1 / 2$ & $2 / 9$ & $3 / 2$ & $1 / 2$ & $-1 / 9$ & $-1 / 2$ & 10 & 2.70 & $1 / 2$ & $\frac{1}{6} \varepsilon_{n}$ \\
\hline $1 / 3$ & $1 / 2$ & $5 / 6$ & $2 / 3$ & $1 / 2$ & $1 / 8$ & $-1 / 4$ & $-1 / 2$ & 6 & 2 & 4 & $\frac{1}{6} \varepsilon_{n}$ \\
\hline
\end{tabular}

The discussion at the end of Section 5, on the completeness of solutions, holds for this model as well.

\section{$7 \quad \mathcal{P} \mathcal{T}$ invariant Generalized Swanson Model}

The importance of quantum systems with $\mathcal{P} \mathcal{T}$ symmetry has already been discussed briefly earlier in this work. So in this section we consider a particular case of the non-Hermitian Hamiltonian in (11) which is symmetric under the combined effect of $\mathcal{P} \mathcal{T}$. For $H$ to be invariant under $\mathcal{P} \mathcal{T}$ symmetry, $\mathcal{A}$ and $\mathcal{A}^{\dagger}$ should also be $\mathcal{P} \mathcal{T}$ invariant. For this purpose, following the $\mathcal{P} \mathcal{T}$ transformations in (2), the operators $\mathcal{A}$ and $\mathcal{A}^{\dagger}$ should transform under parity and time reversal as

$$
\mathcal{P}: \mathcal{A}\left(\mathcal{A}^{\dagger}\right) \rightarrow-\mathcal{A}\left(\mathcal{A}^{\dagger}\right) \quad, \quad \mathcal{T}: \mathcal{A}\left(\mathcal{A}^{\dagger}\right) \rightarrow \mathcal{A}\left(\mathcal{A}^{\dagger}\right)
$$

This is possible only if $W(x)$ transforms under $\mathcal{P} \mathcal{T}$ as

$$
(\mathcal{P} \mathcal{T}) W(x)(\mathcal{P} \mathcal{T})^{-1}=-W(x)
$$

Incidentally, the pseudo superpotentials considered in (28) and (47) fail to obey the above condition (59) for non zero $B_{1}$ or $B_{2}$.

\subsection{Model based on Trigonometric Rosen Morse potential with $B_{1}=0$}

If we consider the particular case $B_{1}=0$ in the trigonometric Rosen Morse model,

$$
W(x)=-A_{1} \cot x \quad, \quad A_{1}>0
$$

then, the pseudo superpotential satisfies the condition (59), and the model, in addition to being $\eta$ pseudo-Hermitian, is also $\mathcal{P} \mathcal{T}$ symmetric. In such a case, both $B_{1}$ and $\mu_{1}$ are 
zero. Thus, though the constraints on $\alpha, \beta$ remain unaltered, the columns $B, B_{1}$ and $\mu_{1}$ are absent in Table 1. For the parameter values already discussed above, the potential in (32) assumes the simple form

$$
V(x)=A(A+1) \csc ^{2} x-A^{2}
$$

with energies

$$
\varepsilon_{n}=(A+n)^{2}-A^{2}
$$

Thus the solutions of the eigenvalue equation in (12) are explicitly given by

$$
\psi_{n}(x) \approx(\sin x)^{A+n+\mu_{2}} P_{n}^{(-A-n,-A-n)}(i \cot x)
$$

with energies $E_{n}=\frac{1}{1-\alpha-\beta} \varepsilon_{n}$.

\subsection{Model based on Hyperbolic Rosen Morse potential with $B_{2}=0$}

Analogous to the previous model, for the particular case $B_{2}=0$, both $\mu_{1}$ and $b$ turn out to be zero, and this non-Hermitian model, too, becomes $\mathcal{P} \mathcal{T}$ symmetric. The potential in (48) reduces to

$$
V(x)=-a(a+1) \operatorname{sech}^{2} x+a^{2}
$$

having real energies

$$
\varepsilon_{n}=\left\{-(a-n)^{2}+a^{2}\right\} \quad, \quad n=0,1, \cdots,<a
$$

and solutions

$$
\psi_{n}(x) \approx(\operatorname{sech} x)^{\left(s-\mu_{2}\right)} P_{n}^{(s, s)}(\tanh x)
$$

where

$$
s_{+}=s_{-}=s=a-n
$$

This enables us to find the eigenfunctions and eigenvalues of the original equation in (12).

Once again, the restrictions on $\alpha, \beta$ are the same as before, viz., conditions (18) and (31), but the columns under $b, B_{2}, \mu_{1}$ are missing from Table 2 .

\section{Conclusions :}

To conclude, we have studied a class of pseudo-Hermitian Hamiltonians (not necessarily $\mathcal{P} \mathcal{T}$ symmetric) of the form $H=\mathcal{A}^{\dagger} \mathcal{A}+\alpha \mathcal{A}^{2}+\beta \mathcal{A}^{\dagger 2}$, where $\alpha$ and $\beta$ are real, 
dimensionless constants $(\alpha \neq \beta)$, and $\mathcal{A}^{\dagger}$ and $\mathcal{A}$ are generalized creation and annihilation operators. Incidentally, Swanson studied a similar model [16], although with harmonic oscillator creation and annihilation operators only. Two explicit examples are considered in this work - viz., models based on the trigonometric Rosen-Morse I and the hyperbolic Rosen-Morse II type potentials. It is observed that the eigen energies are real for a certain range of values of the parameters $\alpha, \beta$. A similarity transformation $\rho$, mapping the nonHermitian Hamiltonian $H$ to a Hermitian one $h$, is also obtained. It is observed that $H$ and $h$ share identical energies. Furthermore, the linear operator $H$ is pseudo-Hermitian with respect to the square of the similarity transformation $\eta=\rho^{2}$. This straightforward approach provides us a simple way of determining the similarity transformation $\rho$, the metric operator $\eta$, as well as the corresponding Hermitian Hamiltonian $h$.

As a mathematical interest, one can also start with the pseudo-Hermitian (but non $\mathcal{P} \mathcal{T}$ symmetric) model $H_{1}$, given by $H_{1}=\mathcal{A} \mathcal{A}^{\dagger}+\alpha \mathcal{A}^{2}+\beta \mathcal{A}^{\dagger 2}$, and proceed as shown in this work. This is possible because of the fact that while in the case of the Swanson model $\left[a, a^{\dagger}\right]=$ constant, the commutator of the generalized annihilation and creation operators $\mathcal{A}$ and $\mathcal{A}^{\dagger}$ is quite non trivial : $\left[\mathcal{A}, \mathcal{A}^{\dagger}\right]=2 W^{\prime}(x)$.

It would be interesting to repeat this analysis with non-Hermitian complex potentials.

As an example, one may write equation (47) as $W(x)=A_{2} \tanh x+i \frac{B_{2}}{A_{2}}$. $H$ obtained in this way, is non-Hermitian, complex and $\mathcal{P} \mathcal{T}$ symmetric, and the procedure is valid for such a case as well. Another interesting area of study would be to examine the applicability of this procedure to non-shape invariant exactly solvable potentials, including QES (quasiexactly solvable) and CES (conditionally exactly solvable) ones.

\section{Acknowledgment}

This work was partly supported by SERC, DST, Govt. of India, through the Fast Track Scheme for Young Scientists (DO No. SR / FTP / PS-07 / 2004), to one of the authors (AS). The authors also thank the referees for their valuable comments.

\section{References}

[1] C. M. Bender \& S. Boettcher, Phys. Rev. Lett. 80 (1998) 5243.

C. M. Bender and S. Boettcher, J. Phys. A : Math. Gen. 31 (1998) L273. 
[2] M. Znojil, J. Phys. A : Math. Gen. 33 (2000) 4561.

G. Lévai and M. Znojil, J. Phys. A : Math. Gen. 33 (2000) 7165.

P. Dorey, C. Dunning and R. Tateo, J. Phys. A : Math. Gen. 34 (2001) 5679.

C. M. Bender, S. Boettcher, H. F. Jones, P. N. Meisinger and M. Simsek, Phys. Lett. A 291 (2001) 197.

Z. Ahmed, Phys. Lett A 282 (2001) 343, ibid. Phys. Lett. A 287 (2001) 295.

B. Bagchi and C. Quesne, Phys. Lett. A 273 (2000) 285, ibid. Phys. Lett. A 300 (2002) 18.

C. M. Bender, D. C. Brody and H. F. Jones, Phys. Rev. Lett. 89 (2002) 270401.

C. M. Bender, D. C. Brody and H. F. Jones, Phys. Rev. Lett. 92 (2004) 119902(E).

C. M. Bender and B. Tan, J. Phys. A : Math. Gen. 39 (2006) 1945.

E. Caliceti, F. Cannata and S. Graffi, J. Phys. A : Math. Gen. 39 (2006) 10019, and references therein.

[3] Proc. 1st Int. Workshop on Pseudo-Hermitian Hamiltonians in Quantum Physics Czech. J. Phys. 54 (2004)

Proc. 2nd Int. Workshop on Pseudo-Hermitian Hamiltonians in Quantum Physics Czech. J. Phys. 54 (2004).

Proc. 3rd Int. Workshop on Pseudo-Hermitian Hamiltonians in Quantum Physics Czech. J. Phys. 55 (2005).

Spl. issue on Physics of non-Hermitian operators (PHHQP IV) J. Phys. A : Math. Gen. 39 (2006).

[4] N. Hatano and D. R. Nelson, Phys. Rev. Lett. 77 (1996) 570.

N. Hatano and D. R. Nelson, Phys. Rev. B 56 (1997) 8651.

D. R. Nelson and N. M. Shnerb, Phys. Rev. E 58 (1998) 1383.

[5] Z. Ahmed, J. Phys. A : Math. Gen. 39 (2006) 9965.

[6] J. C. Muga, J. P. Palao, B. Navarro and I. L. Egusquiza, Phys. Rep. 395357 (2004).

[7] Z. Ahmed, C. M. Bender and M. V. Berry, J. Phys. A : Math. Gen. 38 (2005) L267.

[8] Z. Ahmed, C. M. Bender and M. V. Berry, J. Phys. A : Math. Gen. 38 L627 (2005).

[9] A. Khare and U. Sukhatme, Phys. Lett. A 324 (2004) 406.

A. Khare and U. Sukhatme, J. Math. Phys. 38 (2005) 082106.

[10] J. Feinberg, J. Phys. A : Math. Gen. 3910029 (2006) and references therein.

[11] A. Mostafazadeh, J. Math. Phys. 43 (2002) 205.

A. Mostafazadeh, J. Math. Phys. 43 (2002) 2814.

A. Mostafazadeh, J. Math. Phys. 43 (2002) 3944.

A. Mostafazadeh, J. Math. Phys. 44 (2003) 974. 
[12] L. Solombrino, J. Math. Phys. 43 (2002) 5439.

[13] F. G. Scholtz, H. B. Geyer and F. J. W. Hahne, Ann. Phys. 213 (1992) 74.

R. Kretschmer and L. Szymanowski, Phys. Lett. A 325 (2004) 112.

[14] A. Mostafazadeh and A. Batal, J. Phys. A : Math. Gen. 37 (2004) 11645.

[15] E. C. G. Sudarshan, Phys. Rev. 123 (1961) 2183.

[16] M. S. Swanson, Jour. Math. Phys. 45 (2004) 585.

[17] D. P. Musumbu, H. B. Geyer and W. D. Heiss, J. Phys. A : Math. Theor. 40 (2007) F75.

[18] C. Quesne, Swanson's non-Hermitian Hamiltonian and su(1,1): a way towards generalizations, arXiv : 0705.2868 [mathph].

[19] A. N. F. Aleixo, A. B. Balantekin and M. A. Candilo Ribeiro, J. Phys. A : Math. Gen. 33 (2000) 3173.

A. N. F. Aleixo, A. B. Balantekin and M. A. Candilo Ribeiro, J. Phys. A : Math. Gen. 34 (2001) 1109.

[20] F. Cooper, A. Khare and U. Sukhatme, Supersymmetry in Quantum Mechanics, World Scientific, 2001.

[21] A de souza Dutra, Phys. Rev. A 47 R2435 (1993).

G. Junker and P. Roy, Ann. Phys. 270155 (1998).

[22] H. F. Jones, J. Phys. A : Math. Gen. 38 (2005) 1741.

[23] C. F. M. Faria and A. Fring, J. Phys. A : Math. Gen. 39 (2006) 9269.

[24] L. Gendenshtein, JETP Lett. bf 38356 (1983).

[25] I. Abramowitz and I. A. Stegun, Handbook of Mathematical Functions, Dover, New York, 1970. 